\title{
SCREENING FOR DYSLIPIDEMIA AND EXPEDIENCE OF STATIN THERAPY FOR THE CITIZENS OF TRANSCARPATHIA VALLEY REGIONS WITH OVERWEIGHT AND OBESITY
}

\author{
A. V. Kedyk, M. V. Rishko, O. O. Kutsyn \\ UZHHOROD NATIONAL UNIVERSITY, UZHHOROD, UKRAINE
}

Background. The lipid profiles of patients with overweight, obesity and healthy individuals, the citizens of Transcarpathia valley regions were analysed.

Objective. The study was aimed at evaluation of dyslipidaemia frequency in patients with overweight and obesity, determination of expedience of statins prescription.

Methods. All patients were divided into 2 groups: group 1 - patients with overweight; group 2 - patients with obesity of I and II degree. Estimation of lipid profile parameters was conducted by means of spectrophotometric device SIEMENS Dimension RxL Max. Statistical analysis of the data was conducted using Microsoft Excel 2007.

Results. The patients with obesity had higher level of total cholesterol $(6.03 \pm 0.53 \mathrm{mmol} / \mathrm{l})$, lower HDL-C $(1.15 \pm 0.07 \mathrm{mmol} / \mathrm{l})$ and higher level of $L D L-C(4.19 \pm 0.46 \mathrm{mmol} / \mathrm{l})$ compare with overweight patients. In $46 \%$ of patients with overweight, dyslipidaemia was evidenced and required correction, 27\% of them had high CVR and needed statin therapy, 19\% of people with obesity had moderate CVR and didn't need statins. $77 \%$ of obese patients needed lipid correction, 54\% of them with very high and averagely high level of CVR required statin therapy; 23\% of people with obesity had moderate CVR and did not need statins.

Conclusions. In the studied overweight and obese patients, atherogenic dyslipidaemia was established in $46 \%$ and $77 \%$ of cases respectively. Correction of dyslipidaemia with statin was compulsory for $27 \%$ of patients with overweight and for $54 \%$ with obesity.

KEY WORDS: body mass index; overweight; obesity; lipid profile; statins.

\section{Introduction}

In 2000, the WHO first suggested using the term 'epidemic' to distinguish the situation with obesity prevailing in the world; it was recognized that the number of people with overweight and obesity is progressively increasing.

Over the last 35 years the number of obese patients has increased twice, and over the last 10 years it increased by $75 \%$. According to $\mathrm{WHO}$ data in 2003 there were 1.7 billion of the adult population suffering from overweight (BMI $\geq 25$ $\mathrm{kg} / \mathrm{m}^{2}$ ), the statistics in 2014 announced a new data: there were more than 1.9 billion people with overweight and obesity [1].

In 2016 the results of a meta-analysis on the dynamics of BMI in adults of over 18 years old between 1975 and 2014 were published.

Corresponding author: Antonina Kedyk, Department of Hospital Therapy, Uzhhorod National University, 15a Timiryazeva Street, Uzhhorod, 88000

Phone number: +380968844247

E-mail:kedyk.tonja@gmail.com
1698 data on population studies was analysed, which included 19.2 million people from 186 countries. The increase of average BMI from $21.7 \pm 0.4 \mathrm{~kg} / \mathrm{m}^{2}$ to $24.2 \pm 0.2 \mathrm{~kg} / \mathrm{m}^{2}$ for men and from $22.1 \pm 0.4 \mathrm{~kg} / \mathrm{m}^{2}$ to $24.4 \pm 0.2 \mathrm{~kg} / \mathrm{m}^{2}$ for women was proved [2].

The negative effect of obesity on population is established: this pathology correlates with increased morbidity, mortality, quality of life, and is a significant socioeconomic burden for the world [3]. Several studies prove that obesity increases the cost of medical care and worsens the quality of life [4].

To reduce mortality from cardiovascular diseases in people aged under 65, the Council of Europe in 2002 declared a Plan "Heart of Europe", according to which it is necessary to reduce cholesterol level in the population by less than $5.0 \mathrm{mmol} / \mathrm{l}$, lower blood pressure in the population to a level of $<140 / 90 \mathrm{~mm} \mathrm{Hg}$., reduce the number of smokers by $1 \%$ per 1 year. Thus, by 2020 the mortality rate should de- 
crease by $40 \%$ (2.4\% per year). To achieve this goal, primary care physicians must reorient to early detection of lipid metabolism disorders, their adequate correction and prevention of atherosclerosis associated with cardiovascular diseases and their complications [5].

In most patients with obesity such lipid disorders are observed: rising of small particles of low density lipoprotein (LDL) in blood plasma $[5,6,7]$, which is often associated with increased levels of triglycerides (TG) and decreased level of high-density lipoprotein cholesterol (HDL cholesterol) [5].

Algorithm development for selection of patients for statins therapy, adequate drug choice and its efficacy control, evaluation of target level of cholesterol are the topical matter for a family doctor today.

However, the questions 'how often subclinical dyslipidaemia in obese patients is revealed', and 'how reasonable the prescription of statins for these patients is' are still open, because of the absence of supply for routine lipid profile determination in the practice of family doctors in Ukraine.

The study was aimed at evaluation of dyslipidaemia frequency in patients with overweight and obesity, determination of the expedience of statins prescription.

\section{Methods}

40 individuals who attended clinics of family medicine in Uzhhorod from October to December 2015 were examined.

We analysed the following data in the examined persons: age, sex, level of total cardiovascular risk (CVR), body mass index (BMI), total cholesterol (TC), high-density lipoprotein cholesterol (HDL-C), low density lipoprotein cholesterol (LDL-C), triglycerides (TG), atherogenic coefficient (AC). Estimation of lipid profile parameters was conducted by means of spectrophotometric device SIEMENS Dimension RxL Max.

The study was approved by the Ethics Committee of the Transcarpathian Regional Clinical Cardiology Dispensary. The patient had to sign the informed consent to involvement in the research.

For diagnosis of the overweight or obese we used a body mass index, which was calculated as the ratio of body weight to height squared $\left(\mathrm{kg} / \mathrm{m}^{2}\right)$. The persons, whose BMI was from 25 to $29.99 \mathrm{~kg} / \mathrm{m}^{2}$ composed the group of patients with overweight. The persons with BMI from 30 to $34.99 \mathrm{~kg} / \mathrm{m}^{2}$ were diagnosed with obesity I degree, in the persons with BMI from 35 to $39.99 \mathrm{~kg} / \mathrm{m}^{2}$ obesity II degree was established. The results of lipid profile were interpreted according to guidelines of the ESC and target lipid levels of patients with different CVR.

All patients were divided into 2 groups: group 1 - patients with overweight; group 2 patients with obesity of I and II degree (hereinafter patients with obesity). The share of patients with overweight was $55 \%$ (22 people), with obesity $-45 \%$ (18 persons). Due to the terms and conditions of the research, only those patients, who did not take statins for at least one month before the date of screening, took part in the study.

Patients' data was normally distributed. Statistical analysis of the data: the T-test and Pearson rank correlation coefficient, was conducted using Microsoft Excel 2007.

\section{Results}

The average age of group 1 patients was $57.2 \pm 1.2$ years old, BMI $27.06 \pm 0.42 \mathrm{~kg} / \mathrm{m}^{2}$. The average age of group 2patients was $59.7 \pm 1.1$ years old, BMI $35.9 \pm 1.17 \mathrm{~kg} / \mathrm{m}^{2}$.

Patients with obesity had the following differences in lipid spectrum compared with those who were overweight: higher level of total cholesterol $(6.03 \pm 0.53 \mathrm{mmol} / \mathrm{I}$ vs $4.76 \pm 0.23$ $\mathrm{mmol} / \mathrm{l}, \mathrm{p}=0.048)$, lower HDL-C level $(1.15 \pm 0.07$ $\mathrm{mmol} / \mathrm{l}$ and $1.51 \pm 0.12 \mathrm{mmol} / \mathrm{l}, \mathrm{p}=0.02)$ and higher level of LDL-C $(4.19 \pm 0.46 \mathrm{mmol} / \mathrm{l}$ and $2.89 \pm 0.21 \mathrm{mmol} / \mathrm{l}, \mathrm{p}=0.026$ ).

In the group 1, among all, who required correction of lipid spectrum, $19 \%$ of patients had moderate CVR and didn't need statin therapy because the average level of $L D L-C$ was $2.5 \pm 0.13 \mathrm{mmol} / \mathrm{l}$; respectively, $27 \%$ had increased CVR and average level of LDL-C was $3.4 \pm 0.21 \mathrm{mmol} / \mathrm{l}$ and they needed statin therapy.

Among the obese $77 \%$ of patients required lipid correction. $21 \%$ of group 2 patients had a very high average level of CVR because LDL-C level was $4.4 \pm 0.32 \mathrm{mmol} / \mathrm{l}$ and they needed correction of lipid profile with statins; $33 \%$ of patients had high CVR with an average level of LDL cholesterol $3.7 \pm 0.51 \mathrm{mmol} / \mathrm{l}$ and also needed statin therapy; $23 \%$ of patients with obesity had moderate CVR and did not need statins (Fig.1).

In addition to determination of significant differences between different groups, we also conducted correlation analysis of the groups of patients. In group 1 a direct average relationship was revealed between: the level of total 


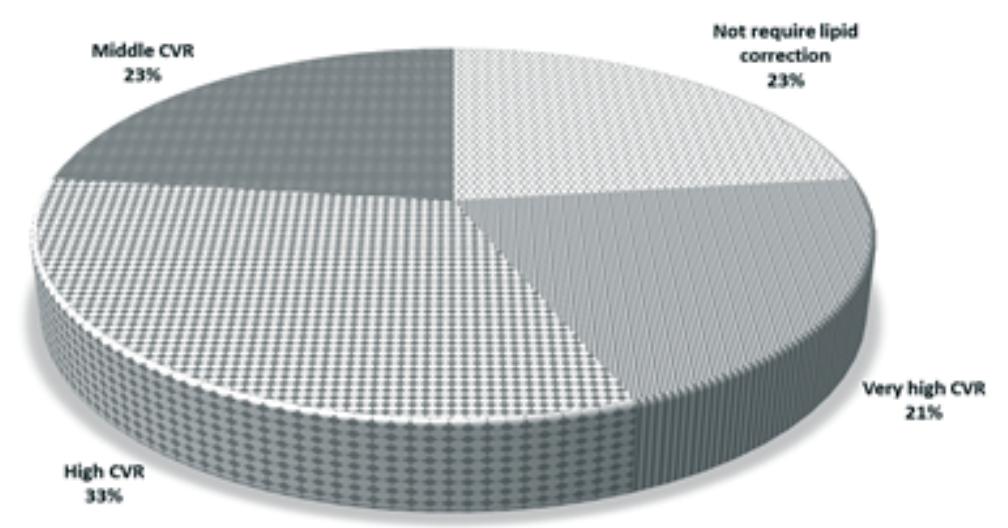

Fig. 1. Percentage of the obese with different cardio-vascular risks and lipid correction is compulsory.

cholesterol and BMI $(r=0.5)$, BMI and LDL cholesterol $(r=0.4)$, BMI and TG ( $r=0.56), B M I$ and atherogenic ratio $(r=0.34)$, inverse middle relationship between age and the level of HDL cholesterol $(r=-0.52)$. Similar correlations were found in the group 2: between BMI and total cholesterol $(r=0.42), B M I$ and LDL cholesterol $(r=0.37), B M I$ and TG $(r=0.46)$.

\section{Discussion}

Thus, with increase of BMI, lipid profile got worse: the levels of total cholesterol and LDL cholesterol increased and of HDL cholesterol decreased. According to the literature $[8,9,10]$, in patients with overweight and obesity most often such lipid disorders are found: rising of small particles of LDL in blood plasma, which is often associated with increased level of TG and decreased level of HDL-C [6]. According to the Framingham Heart Study about $10 \%$ of patients with overweight have increased blood concentrations of cholesterol by $0.3 \mathrm{mmol} / \mathrm{l}$. In young patients with overweight the increased level of cholesterol was twice more than in people of the same age with normal weight. According to the results of population survey conducted in the National Scientific Center "M.D. Strazhesko Institute of Cardiology" MAS of Ukraine; the occurrence of hypercholesterolemia in patients with overweight is $65 \%, 70 \%$ in the obese, and only $40 \%$ in people with normal weight [10].

The method of dyslipidaemia correction was chosen according to the recommendations of ESC/AHA and Association of Cardiologists of Ukraine.

$46 \%$ of patients with overweight had dyslipidaemia, which required correction: lifestyle modification in $19 \%$ and/or statin therapy in $27 \%$.

Thus, with BMI levels increase the levels of total cholesterol, LDL-C and TG significantly increase, in addition in patients of the group 1 HDL-C level decreased with age.

\section{Conclusions}

In the examined overweight and obesity patients, atherogenic dyslipidaemia was established in $46 \%$ and $77 \%$ of cases respectively.

Correction of dyslipidaemia by statin was compulsory for $27 \%$ of patients with overweight and $54 \%$ with obesity.

Regardless of gender and age, in the patients with increased BMI level, the increased levels of total cholesterol, LDL cholesterol and atherogenic factor were observed.

\section{References}

1. Obesity: world statistic. 2015. https://medaboutme.ru/zdorove/publikacii/stati/sovety_vracha/ ozhirenie_mirovaya_statistika/?utm_source=copypaste\&utm_medium=referral\&utm_campaign=copypaste? utm_source=copypaste\&utm_medium= referral\&utm_campaign=copypaste. Accessed 31 June 2015.

2. NCD Risk Factor Collaboration (Mariachiara Di Cesare, James Bentham, Gretchen A Stevens and other more than 150 authors). Trends in adult body- 
mass index in 200 countries from 1975 to 2014: a pooled analysis of 1698 population-based measurement studies with $19 \cdot 2$ million participants. Lancet. 2016;387:1377-96.

3. Frühbeck G, Toplak H, Woodward E, Yumuk V, Maislos M, Oppert JM. Obesity: The Gateway to ill Health - an EASO position statement on a rising public health, clinical and scientific challenge in Europe. Obes Facts. 2013;6:117-120.

4. Zhang J, Shi XM, Liang XF. Economic costs of both overweight and obesity among Chinese urban and rural residents, in 2010. Zhonghua Liu Xing Bing XueZaZhi. 2013;34(6):598-600.

5. Magkos F, Mohammed BS, Mittendorfer B. Effect of obesity on the plasma lipoprotein subclass profile in normoglycemic and normolipidemic men and women. Int J Obes. 2008;32(11):1655-1664.
6. Rosolova H, Nussbaumerova B. Cardio-metabolic risk prediction should be superior to cardiovascular risk assessment in primary prevention of cardiovascular diseases. EPMA J. 2011;2(1):15-26.

7. Rizzo M, Mikhailidis DP. There is more to predicting vascular disease than just established risk factors. Curr Pharm Des. 2011;17(33):3608-3610.

8. Tvorogova M.G. Reverse cholesterol transport. Cardiology 2011;2:66-70.

9. Rizzo M, Kotur-Stevuljevic J, Berneis K, Spinas G, Rini GB, Jelic-Ivanovic Z, et al. Atherogenic dyslipidaemia and oxidative stress: A new look Transl Res. 2009;153(5):217-223.

10. Sokolova EI. Decrease of the importance of antiatherogenic high-density lipoproteins in obese. Cardilogy. 2010;2:45-50. 